\title{
's why Selenium-Ligated Palladium (II) Complexes as Highly Active Catalysts for Carbon-Carbon Coupling Reactions: the Heck Reaction
}

\author{
Qingwei Yao,* Elizabeth P. Kinney, and Chong Zheng \\ Department of Chemistry and Biochemistry, The Michael Faraday Laboratories, \\ Northern Illinois University, \\ DeKalb, Illinois 60115
}

General. All aryl halides(Acros, Aldrich, Fisher, Lancaster), olefins (Aldrich), N,Ndimethylacetamide (anhydrous; Sure-Seal, Aldrich) were used as received from commercial sources. Triethylamine (Fisher) was distilled under argon from sodium. Benzene and THF were distilled from sodium/benzophenone under a nitrogen atmosphere prior to use. Sodium carbonate (Fisher), sodium acetate (Aldrich), potassium carbonate (Aldrich), potassium acetate (Aldrich) and potassium phosphate (Aldrich) were ground into a fine powder and stored in sealed vials. Palladium(II) acetate, bis(benzonitrile)dichloropalladium(II) and di- $\mu$-chlorobis[2[(dimethylamino)methyl]phenyl- $C-N]$ dipalladium were purchased from Aldrich and used as received. Flash chromatography was performed on silica gel 60 (230-400 mesh). Unless otherwise noted, NMR spectra were acquired at $25{ }^{\circ} \mathrm{C}$ in $\mathrm{CDCl}_{3}$ at $500 \mathrm{MHz}$ for ${ }^{1} \mathrm{H}$ and 125 $\mathrm{MHz}$ for ${ }^{13} \mathrm{C}$. All reactions were carried out under an argon atmosphere in oven-dried Schlenk flasks.

\section{Synthesis of Pd-complex 1}

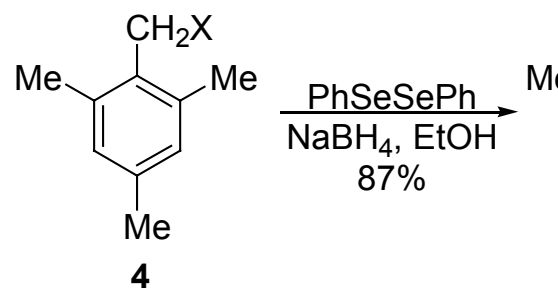<smiles>Cc1cc(C)c(C)c([AsH2])c1</smiles>
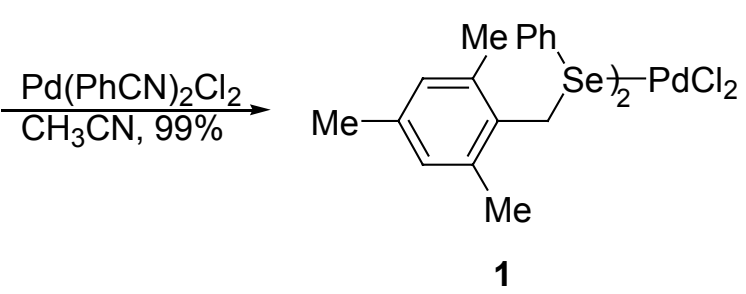

An oven-dried flask was charged with diphenyl diselenide $(312 \mathrm{mg}, 1.0 \mathrm{mmol})$ and absolute ethanol $(20.0 \mathrm{~mL}) . \mathrm{NaBH}_{4}(84 \mathrm{mg}, 2.2 \mathrm{mmol})$ was then added in portions. After stirring for 10 min at rt, 2,4,6-trimethylbenzyl chloride (4) (340 mg, $2.0 \mathrm{mmol}$ ) was added in one portion. After stirring at $\mathrm{rt}$ for $2 \mathrm{~h}$, the reaction was quenched with water $(100 \mathrm{~mL})$ and extracted with ethyl 
acetate $(3 \times 40 \mathrm{~mL})$. The combined organic extracts were washed with brine, dried $\left(\mathrm{Na}_{2} \mathrm{SO}_{2}\right)$ and concentration to dryness. Purification by flash column chromatography on silica gel (hexanes $\left./ \mathrm{CH}_{2} \mathrm{Cl}_{2} 3: 1\right)$ gave $5\left(505 \mathrm{mg}, 87 \%\right.$ ) as a pale yellow oil. ${ }^{1} \mathrm{H} \mathrm{NMR}\left(500 \mathrm{MHz}, \mathrm{CDCl}_{3}\right): \delta$ $7.56-7.55(\mathrm{~m}, 2 \mathrm{H}), 7.30-7.28(\mathrm{~m}, 3 \mathrm{H}), 6.86(\mathrm{~s}, 2 \mathrm{H}), 4.21(\mathrm{~s}, 2 \mathrm{H}), 2.35$ (s, $6 \mathrm{H}), 2.29$ (s, $3 \mathrm{H})$. ${ }^{13} \mathrm{C}$ NMR (125 MHz, $\mathrm{CDCl}_{3}$ at $\left.25{ }^{\circ} \mathrm{C}\right): \delta 136.9,136.5,133.4,131.3,131.1,129.0$ (2 C's), 127.1, 27.2, 21.0, 19.6). ${ }^{13} \mathrm{C} \mathrm{NMR}\left(125 \mathrm{MHz}, \mathrm{CDCl}_{3}\right.$ at $\left.50{ }^{\circ} \mathrm{C}\right): \delta 136.80,136.40,133.52,131.23$, 131.16, 128.99, 128.90, 127.07, 27.23, 20.82, 19.48. Anal. Calcd for $\mathrm{C}_{16} \mathrm{H}_{18} \mathrm{Se}: \mathrm{C}, 66.43$; H, 6.27. Found: C, 66.53; H, 6.27.

An oven-dried flask was charged with $5(282 \mathrm{mg}, 0.98 \mathrm{mmol})$ and $\mathrm{Pd}(\mathrm{PhCN})_{2} \mathrm{Cl}_{2}(187 \mathrm{mg}$, $0.49 \mathrm{mmol}) . \mathrm{CH}_{3} \mathrm{CN}(3.0 \mathrm{~mL})$ was then added via syringe. The reaction mixture was stirred at $\mathrm{rt}$ for $1.5 \mathrm{~h}$ and then concentrated to dryness under vacuum. The crude reaction product was dissolved in small amount of $\mathrm{CH}_{2} \mathrm{Cl}_{2}$ and triturated with hexanes. After filtration and drying 1 (365 mg, 99 \%) was obtained as an orange red solid: m.p. $135-137{ }^{\circ} \mathrm{C}$. Crystals suitable for Xray diffraction analysis were obtained by laying hexanes on top of a solution of 1 in $\mathrm{CH}_{2} \mathrm{Cl}_{2}$. ${ }^{1} \mathrm{H}$ NMR (500 MHz, $\left.\mathrm{CDCl}_{3}\right):{ }^{1} \mathrm{H}$ NMR $\left(500 \mathrm{MHz}, \mathrm{CDCl}_{3}\right): \delta 7.86(\mathrm{~d}, 4 \mathrm{H}, J=7.5 \mathrm{~Hz}), 7.45$ (t, $2 \mathrm{H}$, $J=7.5 \mathrm{~Hz}), 7.32$ (t, $4 \mathrm{H}, J=7.6 \mathrm{~Hz}), 6.78$ (s, $4 \mathrm{H}), 4.62(\mathrm{br}, 4 \mathrm{H}), 2.26(\mathrm{~s}, 6 \mathrm{H}), 2.06$ (s, $12 \mathrm{H})$. $\delta{ }^{13} \mathrm{C}$ NMR (125 MHz, $\left.\mathrm{CDCl}_{3}\right): \delta 137.9,137.8,135.0,130.1,129.2,129.2,128.1,127.6,35.3$, 21.0, 20.1. Anal. Calcd. For $\mathrm{C}_{32} \mathrm{H}_{36} \mathrm{Cl}_{2} \mathrm{PdSe}_{2}$ : C, 50.85; H, 4.80. Found: C, 50.70; H, 4.94.

\section{Synthesis of Pd-complex 2}

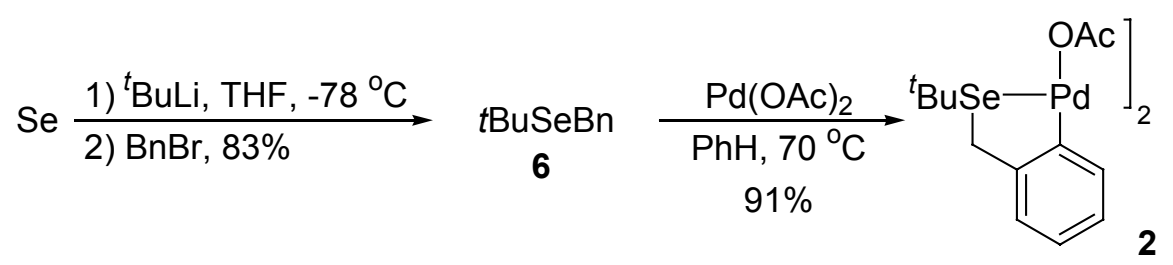

An oven-dried flask was charged with selenium powder (398 mg, $5 \mathrm{mmol})$ and THF (25 mL) under argon. The suspension was cooled to $-78{ }^{\circ} \mathrm{C}$ and tert-butyllithium $(3.5 \mathrm{~mL}, 1.7 \mathrm{M}, 6.0$ mmol) was added dropwise with stirring via syringe. The reaction mixture was stirred until a homogeneous orange solution formed. Benzyl bromide $(600 \mu \mathrm{L}, 5 \mathrm{mmol})$ was then added. The reaction was gradually warmed to $\mathrm{rt}$ and stirred for $5 \mathrm{~h}$. Water $(100 \mathrm{~mL})$ was then added and the reaction mixture was extracted with ethyl acetate $(3 \times 75 \mathrm{~mL})$. The combined organic extracts were washed with brine, dried over $\mathrm{Na}_{2} \mathrm{SO}_{2}$ and concentration. Purification by flash column chromatography on silica gel (hexanes $\left./ \mathrm{CH}_{2} \mathrm{Cl}_{2} 1: 1\right)$ gave $6(944 \mathrm{mg}, 83 \%)$ as a pale yellow oil. 
${ }^{1} \mathrm{H}$ NMR (500 MHz, $\left.\mathrm{CDCl}_{3}\right): \delta 7.37$ (d, 2 H. $\left.J=7.5 \mathrm{~Hz}\right), 7.31(\mathrm{t}, 2 \mathrm{H}, J=7.5 \mathrm{~Hz}), 7.22(\mathrm{t}, 1 \mathrm{H}, J$ $=7.5 \mathrm{~Hz}), 3.89(\mathrm{~s}, 2 \mathrm{H}), 1.52(\mathrm{~s}, 9 \mathrm{H}) .{ }^{13} \mathrm{C} \mathrm{NMR}\left(125 \mathrm{MHz}, \mathrm{CDCl}_{3}\right): \delta 139.3,129.0,128.5$, 126.5, 40.1, 32.4, 26.07. Anal. Calcd for $\mathrm{C}_{11} \mathrm{H}_{16} \mathrm{Se}$ : C, 58.15; H, 7.10. Found: C, 58.32; H, 7.31.

A flame-dried flask was charged under Ar with $\mathrm{Pd}(\mathrm{OAc})_{2}(46 \mathrm{mg}, 0.205 \mathrm{mmol})$ and 5 (45.4 $\mathrm{mg}, 0.20 \mathrm{mmol})$. Benzene $(4.0 \mathrm{~mL})$ was then added via syringe and the reaction mixture was stirred for $5 \mathrm{~min}$ at $\mathrm{rt}$. The orange red solution was then heated to $70{ }^{\circ} \mathrm{C}$ for $1 \mathrm{~h}$. After cooling to $\mathrm{rt}$, the reaction mixture was taken to dryness under vacuum. The crude reaction mixture was dissolved in small amount $(\sim 0.3 \mathrm{~mL})$ of $\mathrm{CH}_{2} \mathrm{Cl}_{2}$ and diluted with hexanes. The insoluble residues were then removed by filtration and the clear solution was concentrated under reduced pressure and dried to give $2(71 \mathrm{mg}, 91 \%)$ as an orange yellow foam. ${ }^{1} \mathrm{H}$ NMR (500 MHz, $\left.\mathrm{CDCl}_{3}\right): \delta 7.38(\mathrm{dd}, 1 \mathrm{H}, J=1.2$ and $7.8 \mathrm{~Hz}), 6.93(\mathrm{dt}, 1 \mathrm{H}, J=1.4$ and $7.3 \mathrm{~Hz}), 6.89(\mathrm{dt}, 1 \mathrm{H}, J=$ 1.7 and $7.4 \mathrm{~Hz}), 6.76(\mathrm{bd}, 1 \mathrm{H}, J=7.1 \mathrm{~Hz}), 3.33(\mathrm{~d}, 1 \mathrm{H}, J=15.1 \mathrm{~Hz}), 2.90$ (d, $1 \mathrm{H}, J=15.0 \mathrm{~Hz})$, $2.14(\mathrm{~s}, 3 \mathrm{H}), 1.46(\mathrm{~s}, 9 \mathrm{H}) .{ }^{13} \mathrm{C} \mathrm{NMR}\left(125 \mathrm{MHz}, \mathrm{CDCl}_{3}\right): \delta 180.3,152.0,143.9,135.1,124.8$, 124.2, 123.3, 49.1, 31.4, 31.1, 24.6.

\section{Synthesis of Pd-complex 3}

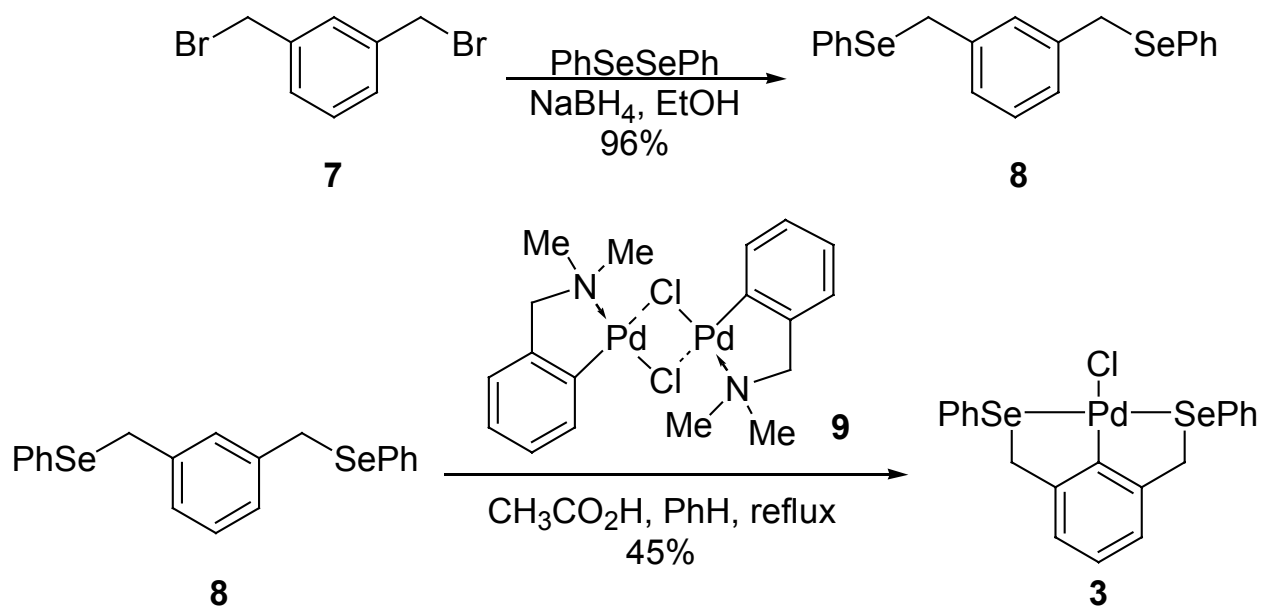

An oven-dried flask was charged with diphenyl diselenide $(313 \mathrm{mg}, 1.0 \mathrm{mmol})$ and absolute ethanol $(15.0 \mathrm{~mL}) . \mathrm{NaBH}_{4}(80 \mathrm{mg}, 2.1 \mathrm{mmol})$ was then added in portions. After stirring at rt for $20 \mathrm{~min}, \alpha, \alpha^{\prime}$-dibromo- $m$-xylene $7(264 \mathrm{mg}, 1.0 \mathrm{mmol})$ was added in one portion and the reaction mixture was stirred at $\mathrm{rt}$ for $1 \mathrm{~h}$. Water $(100 \mathrm{~mL})$ was then added and the reaction mixture extracted with ethyl acetate $(3 \times 40 \mathrm{~mL})$. The combined organic extracts were washed with brine, dried over $\left(\mathrm{Na}_{2} \mathrm{SO}_{2}\right)$ and concentration to dryness. Purification by flash column chromatography on silica gel (hexanes/ $\mathrm{CH}_{2} \mathrm{Cl}_{2} 3: 1$ ) gave 8 (398 mg, $96 \%$ ) as a slightly yellow 
solid. ${ }^{1} \mathrm{H}$ NMR (500 MHz, $\left.\mathrm{CDCl}_{3}\right): \delta 7.44$ - 7.46 (m, $\left.4 \mathrm{H}\right), 7.24-7.30(\mathrm{~m}, 6 \mathrm{H}), 7.14(\mathrm{~m}, 1 \mathrm{H})$, $7.04-7.06(\mathrm{~m}, 3 \mathrm{H}), 4.06(\mathrm{~s}, 4 \mathrm{H}) .{ }^{13} \mathrm{C} \mathrm{NMR}\left(125 \mathrm{MHz}, \mathrm{CDCl}_{3}\right): \delta 138.8,133.6,130.3,129.4$, 129.0, 128.5, 127.4. Anal. Calcd for $\mathrm{C}_{20} \mathrm{H}_{18} \mathrm{Se}_{2}$ : C, 57.71; H, 4.36. Found: C, 57.63; H, 4.32.

An oven-dried flask was charged with 8 (109 mg, $0.26 \mathrm{mmol})$ and di- $\mu$-chlorobis[2-[(dimethylamino)methyl]phenyl-C,N]dipalladium (70 mg, $0.125 \mathrm{mmol})$. Glacial acetic acid (85 $\mu \mathrm{L})$ was then added via syringe followed by benzene $(3.5 \mathrm{~mL})$. The reaction mixture was heated to reflux for $3.5 \mathrm{~h}$ before cooling to $\mathrm{rt}$. After taken to dryness under vacuum, the crude reaction mixture was washed with hexanes $(3 \mathrm{x} 4 \mathrm{~mL})$. The residue was then dissolved in $\mathrm{CH}_{2} \mathrm{Cl}_{2}(2 \mathrm{~mL})$ and hexanes $(3 \mathrm{~mL})$ were added. Further purification by drying under vacuum gave the orange brown solid $3(65 \mathrm{mg}, 45 \%)$ as a diastereomeric mixture (ratio 1:1.1). ${ }^{1} \mathrm{H}$ NMR (500 MHz, $\left.\mathrm{CDCl}_{3}\right): \delta 7.90(\mathrm{t}, 4 \mathrm{H}, J=5.9 \mathrm{~Hz}), 7.41-7.32(\mathrm{~m}, 6 \mathrm{H}), 6.95(\mathrm{~m}, 3 \mathrm{H}) .4 .73(\mathrm{~d}, J=14.9 \mathrm{~Hz}$, major diastereomer) and $4.72(\mathrm{~d}, \mathrm{~J}=14.6 \mathrm{~Hz}$, minor diastereomer) $(1 \mathrm{H}$ in total), $4.32(\mathrm{~d}, J=$ $14.8 \mathrm{~Hz}$, minor diastereomer) and $4.27(\mathrm{~d}, \mathrm{~J}=15.0 \mathrm{~Hz})(1 \mathrm{H}$ in total $) .{ }^{13} \mathrm{C} \mathrm{NMR}(125 \mathrm{MHz}$, $\left.\mathrm{CDCl}_{3}\right) \delta 157.5$ and $157.11,151.2$ and 150.8, 132.9 and 132.6, 129.8, 129.72 and 129.5, 129.2 and 129.0, 124.3 and 124.1, 123.8, 43.2 and 42.8. Anal Calcd. For $\mathrm{C}_{20} \mathrm{H}_{17} \mathrm{ClPdSe}_{2}: \mathrm{C}, 43.12 ; \mathrm{H}$, 3.08. Found C, $43.01 ; \mathrm{H}, 3.37$.

The Heck reaction of bromobenzene with $\boldsymbol{n}$-butyl acrylate in the presence of catalyst 1 (Table 1, entry 1). General Procedure for the Heck Reaction Using Catalyst 1:

An oven-dried Schlenk flask was charged under Ar with bromobenzene (525 $\mu \mathrm{L}, 784 \mathrm{mg}, 5.0$ mmol), $n$-butyl acrylate $(850 \mu \mathrm{L}, 6.0 \mathrm{mmol})$, anhydrous $\mathrm{Na}_{2} \mathrm{CO}_{3}(594 \mathrm{mg}, 5.6 \mathrm{mmol})$ and DMA (4.9 mL). A solution of catalyst 1 in DMA $\left(2.5 \times 10^{-4} \mathrm{M}, 140 \mu \mathrm{L}, 3.5 \times 10^{-5} \mathrm{mmol}, 7.0 \times 10^{-4} \mathrm{~mol}\right.$ $\%$ ) was then added via syringe. The flask was sealed and placed in a $140{ }^{\circ} \mathrm{C}$ oil bath controlled by a J-KEMP temperature controller and stirred for $24 \mathrm{~h}$. After cooling to $\mathrm{rt}$, the reaction mixture was poured into water $(50 \mathrm{~mL})$ and extracted with EtOAc $(3 \times 40 \mathrm{~mL})$. The combined organic extracts were washed with brine, dried $\left(\mathrm{Na}_{2} \mathrm{SO}_{4}\right)$ and concentrated. Purification by flash chromatography on silica gel (hexanes/ $\mathrm{CH}_{2} \mathrm{Cl}_{2} 1: 1$ ) gave pure $n$-butyl trans-cinnamate (349 mg, $34.2 \%)$. In another parallel run under identical conditions, the reaction gave $351 \mathrm{mg}(34.6 \%)$ of the product after workup and purification.

The Heck reaction of bromobenzene with $\boldsymbol{n}$-butyl acrylate in the presence of catalyst 2 (Table 1, entry 5). General Procedure for the Heck Reaction Using Catalyst 2: 
An oven dried Schlenk flask was charged under Ar with bromobenzene (525 $\mu \mathrm{L}, 784 \mathrm{mg}, 5.0$ mmol), $n$-butyl acrylate $(850 \mu \mathrm{L}, 6.0 \mathrm{mmol})$, anhydrous $\mathrm{Na}_{2} \mathrm{CO}_{3}(595 \mathrm{mg}, 5.6 \mathrm{mmol})$ and DMA $(5.0 \mathrm{~mL})$. A solution of catalyst 2 in DMA $\left(1.5 \times 10^{-3} \mathrm{M}, 45 \mu \mathrm{L}, 6.65 \times 10^{-5} \mathrm{mmol}, 1.35 \times 10^{-3}\right.$ mol \%) was then added via syringe. The flask was sealed and placed in a $140{ }^{\circ} \mathrm{C}$ oil bath controlled by a J-KEMP temperature controller and stirred for $24 \mathrm{~h}$. After cooling to $\mathrm{rt}$, the reaction mixture was poured into water $(50 \mathrm{~mL})$ and extracted with EtOAc $(3 \mathrm{x} 40 \mathrm{~mL})$. The combined organic extracts were washed with brine, dried $\left(\mathrm{Na}_{2} \mathrm{SO}_{4}\right)$ and concentrated. Purification by flash chromatography on silica gel (hexanes $/ \mathrm{CH}_{2} \mathrm{Cl}_{2} 1: 1$ ) gave pure $n$-butyl transcinnamate (709 mg, $69.4 \%$ ). In another parallel run under identical conditions, the reaction gave $740 \mathrm{mg}(72.5 \%)$ of the product after workup and purification.

The Heck reaction of bromobenzene with $n$-butyl acrylate in the presence of catalyst 3 (Table 1, entry 9). General Procedure for the Heck Reaction Using Catalyst 3:

An oven-dried Schlenk flask was charged under Ar with bromobenzene (525 $\mu \mathrm{L}, 783 \mathrm{mg}, 5.0$ mmol), $n$-butyl acrylate $(850 \mu \mathrm{L}, 6.0 \mathrm{mmol})$, anhydrous $\mathrm{Na}_{2} \mathrm{CO}_{3}(594 \mathrm{mg}, 5.6 \mathrm{mmol})$ and DMA $(5.0 \mathrm{~mL})$. A solution of catalyst 3 in DMA $\left(7.9 \times 10^{-4} \mathrm{M}, 44 \mu \mathrm{L}, 3.5 \times 10^{-5} \mathrm{mmol}, 7.0 \times 10^{-4} \mathrm{~mol}\right.$ $\%$ ) was then added via syringe. The flask was sealed and placed in a $140{ }^{\circ} \mathrm{C}$ oil bath controlled by a J-KEMP temperature controller and stirred for $24 \mathrm{~h}$. After cooling to $\mathrm{rt}$, the reaction mixture was poured into water $(50 \mathrm{~mL})$ and extracted with EtOAc $(3 \times 40 \mathrm{~mL})$. The combined organic extracts were washed with brine, dried $\left(\mathrm{Na}_{2} \mathrm{SO}_{4}\right)$ and concentrated. Purification by flash chromatography on silica gel (hexanes $/ \mathrm{CH}_{2} \mathrm{Cl}_{2} 1: 1$ ) gave pure $n$-butyl trans-cinnamate (508 $\mathrm{mg}$, $49.7 \%)$. In another parallel run under identical conditions, the reaction gave $521 \mathrm{mg}(51.0 \%)$ of the product after workup and purification.

The Heck reaction of bromobenzene with styrene in the presence of catalyst 2 (Table 2 , entry 3). General Procedure for the Heck Reaction of Aryl Bromides with Styrene Using Catalyst 2:

An oven-dried Schlenk flask was charged under Ar with bromobenzene (420 $\mu \mathrm{L}, 626 \mathrm{mg}, 4.0$ $\mathrm{mmol})$, styrene $(560 \mu \mathrm{L}, 508 \mathrm{mg}, 4.8 \mathrm{mmol})$, sodium acetate $(394 \mathrm{mg}, 4.8 \mathrm{mmol})$ and DMA (4.0 $\mathrm{mL})$. A solution of catalyst 2 in DMA $\left(1.5 \times 10^{-3} \mathrm{M}, 52 \mu \mathrm{L}, 7.8 \times 10^{-4} \mathrm{mmol}, 2.0 \times 10^{-3} \mathrm{~mol} \%\right)$ was then added via syringe. The flask was sealed and placed in a $140^{\circ} \mathrm{C}$ oil bath controlled by a J-KEMP temperature controller and stirred for $20 \mathrm{~h}$. After cooling to rt, the reaction was quenched by water $(50 \mathrm{~mL})$ and extracted ethyl acetate $(3 \times 40 \mathrm{~mL})$. The combined organic 
extracts were washed with brine, dried over $\mathrm{Na}_{2} \mathrm{SO}_{2}$ and concentration to dryness. Purification by flash column chromatography on silica gel (hexanes $/ \mathrm{CH}_{2} \mathrm{Cl}_{2} 1: 1$ ) to give pure trans-stilbene (704 mg, 97.6 \%). In another parallel run under identical conditions, the reaction gave $699 \mathrm{mg}$ (97\%) of the product after workup and purification.

\section{${ }^{1}$ H NMR Data of Isolated Pure Products.}

All of the Heck reaction products of Tables 1 and 2 are known compounds and were isolated by rigorous chromatographic purification. The identity and purity of the isolated products were secured by $500{ }^{1} \mathrm{H}$ NMR spectroscopy analysis as listed below.

trans-Cinnamic acid $n$-butyl ester<smiles>CCCCOC(=O)/C=C/c1ccccc1</smiles>

${ }^{1} \mathrm{H}$ NMR (500 MHz, $\left.\mathrm{CDCl}_{3}\right): \delta 7.71(\mathrm{~d}, 1 \mathrm{H}, \mathrm{J}=16.0 \mathrm{~Hz}), 7.55(\mathrm{~m}, 2 \mathrm{H}), 7.41(\mathrm{~m}, 3 \mathrm{H}), 6.47$ (d, $1 \mathrm{H}, J=16.0 \mathrm{~Hz}$ ), 4.24 (t, $2 \mathrm{H}, J=6.7 \mathrm{~Hz}$ ), 1.72 (quint, $2 \mathrm{H}, J=7.2 \mathrm{~Hz}$ ), 1.47 (sixet, $2 \mathrm{H}, J=7.5$ $\mathrm{Hz}), 0.99$ (t, 3H, $J=7.4 \mathrm{~Hz})$.

\section{trans-4-Methylcinnamic acid $n$-butyl ester}<smiles>CCCCOC(=O)/C=C/c1ccc(C)cc1</smiles>

${ }^{1} \mathrm{H}$ NMR $\left(500 \mathrm{MHz}, \mathrm{CDCl}_{3}\right): \delta 7.68(\mathrm{~d}, 1 \mathrm{H}, J=16.0 \mathrm{~Hz}), 7.45(\mathrm{~d}, 2 \mathrm{H}, J=8.1 \mathrm{~Hz}), 7.22(\mathrm{~d}, 2 \mathrm{H}$, $J=7.9 \mathrm{~Hz}), 6.42(\mathrm{~d}, 1 \mathrm{H}, J=16.0 \mathrm{~Hz}), 4.23(\mathrm{t}, 2 \mathrm{H}, J=6.7 \mathrm{~Hz}), 2.40(\mathrm{~s}, 3 \mathrm{H}), 1.72$ (quint, $2 \mathrm{H}, J$ $=7.1 \mathrm{~Hz}$ ), 1.46 (sixet, $2 \mathrm{H}, J=7.5 \mathrm{~Hz}), 0.99$ (t, $3 \mathrm{H}, J=7.4 \mathrm{~Hz}$ ),

trans-4-Methoxycinnamic acid $n$-butyl ester<smiles>CCCCOC(=O)/C=C/c1ccc(OC)cc1</smiles>

${ }^{1} \mathrm{H}$ NMR $\left(500 \mathrm{MHz}, \mathrm{CDCl}_{3}\right): \delta 7.66(\mathrm{~d}, 1 \mathrm{H}, J=16.0 \mathrm{~Hz}), 7.50(\mathrm{~d}, 2 \mathrm{H}, J=8.7 \mathrm{~Hz}), 6.92(\mathrm{~d}, 2 \mathrm{H}$, $J=8.7 \mathrm{~Hz}), 6.34(\mathrm{~d}, 1 \mathrm{H}, J=16.0 \mathrm{~Hz}), 4.22(\mathrm{t}, 2 \mathrm{H}, J=6.7 \mathrm{~Hz}), 3.86$ (s, $3 \mathrm{H}), 1.71$ (quint, $2 \mathrm{H}, J$ $=7.1 \mathrm{~Hz}$ ), 1.46 (sixet, $2 \mathrm{H}, J=7.5 \mathrm{~Hz}$ ), 0.99 (t, $3 \mathrm{H}, J=7.4 \mathrm{~Hz}$ ). 
<smiles>CCCCOC(=O)/C=C/c1ccc(C=O)cc1</smiles>

${ }^{1} \mathrm{H}$ NMR (500 MHz, $\mathrm{CDCl}_{3}$ ): $\delta 10.03$ (s, $\left.1 \mathrm{H}\right), 7.90$ (d, $\left.2 \mathrm{H}, J=8.2 \mathrm{~Hz}\right), 7.71(\mathrm{~d}, 1 \mathrm{H}, J=16.2$ Hz), $7.68(\mathrm{~d}, 2 \mathrm{H}, J=8.2 \mathrm{~Hz}), 6.55(\mathrm{~d}, 1 \mathrm{H}, J=16.1 \mathrm{~Hz}), 4.23(\mathrm{t}, 2 \mathrm{H}, J=6.9 \mathrm{~Hz}), 1.70(\mathrm{~m}, 2 \mathrm{H})$, $1.45(\mathrm{~m}, 2 \mathrm{H}), 0.97$ (t, $3 \mathrm{H}, J=7.4 \mathrm{~Hz})$.

trans-4-Cyanocinnamic acid $n$-butyl ester<smiles>CCC(C)OC(=O)/C=C/c1ccc(C#N)cc1</smiles>

${ }^{1} \mathrm{H}$ NMR (500 MHz, $\left.\mathrm{CDCl}_{3}\right): \delta 7.70(\mathrm{~d}, 2 \mathrm{H}, J=8.0 \mathrm{~Hz}), 7.68(\mathrm{~d}, 1 \mathrm{H}, J=16.4 \mathrm{~Hz}), 7.63(\mathrm{~d}, 2 \mathrm{H}$, $J=8.2 \mathrm{~Hz}), 6.54(\mathrm{~d}, 1 \mathrm{H}, J=16.1 \mathrm{~Hz}), 4.25$ (t, $2 \mathrm{H}, J=6.7 \mathrm{~Hz}), 1.72$ (quint, $2 \mathrm{H}, J=7.1 \mathrm{~Hz}$ ), 1.46 (sixet, $2 \mathrm{H}, J=7.4 \mathrm{~Hz}$ ), 0.99 (t, $3 \mathrm{H}, J=7.4 \mathrm{~Hz}$ ).

\section{trans-4-(Trifluoromethyl)cinnamic acid $\boldsymbol{n}$-butyl ester}<smiles>CCC(C)OC(=O)/C=C/c1ccc(C(F)(F)F)cc1</smiles>

${ }^{1} \mathrm{H}$ NMR $\left(500 \mathrm{MHz}, \mathrm{CDCl}_{3}\right): \delta 7.71(\mathrm{~d}, 1 \mathrm{H}, J=16.1 \mathrm{~Hz}), 7.67(\mathrm{~d}, 2 \mathrm{H}, J=8.6 \mathrm{~Hz}), 7.65(\mathrm{~d}, 2 \mathrm{H}$, $J=8.8 \mathrm{~Hz}), 6.54(\mathrm{~d}, 1 \mathrm{H}, J=16.1 \mathrm{~Hz}), 4.25(\mathrm{~d}, 2 \mathrm{H}, J=6.7 \mathrm{~Hz}), 1.72$ (quint, $2 \mathrm{H}, J=7.1 \mathrm{~Hz}$ ), 1.479 (sixet, $2 \mathrm{H}, J=7.5 \mathrm{~Hz}$ ), 1.00 (t, $3 \mathrm{H}, J=7.4 \mathrm{~Hz}$ ).

trans-3-(3-Pyridinyl)acrylic acid $n$-butyl ester<smiles>CCCCOC(=O)/C=C/c1cccnc1</smiles>

${ }^{1} \mathrm{H}$ NMR $\left(500 \mathrm{MHz}, \mathrm{CDCl}_{3}\right): \delta 8.76(\mathrm{~s}, 1 \mathrm{H}), 8.61(\mathrm{~m}, 1 \mathrm{H}), 7.85(\mathrm{~d}, 1 \mathrm{H}, J=7.9 \mathrm{~Hz}), 7.68(1 \mathrm{H}, J$ $=16.1 \mathrm{~Hz}), 7.34(\mathrm{~m}, 1 \mathrm{H}), 6.53(\mathrm{~d}, 1 \mathrm{H}, J=16.1 \mathrm{~Hz}), 4.24(\mathrm{t}, 2 \mathrm{H}, J=6.7 \mathrm{~Hz}), 1.71$ (quint, $2 \mathrm{H}, J$ $=7.2 \mathrm{~Hz}$ ), 1.45 (sixet, $2 \mathrm{H}, J=7.5 \mathrm{~Hz}$ ), 0,98 (t, $3 \mathrm{H}, J=7.4 \mathrm{~Hz}$ ).

trans-3-(2-Thiophenyl)acrylic acid $n$-butyl ester<smiles>CCC(C)OC(=O)/C=C/c1cccs1</smiles> 
${ }^{1} \mathrm{H}$ NMR (500 MHz, CDCl $): \delta 7.79$ (d, $\left.1 \mathrm{H}, J=15.7 \mathrm{~Hz}\right), 7.38(\mathrm{~d}, 1 \mathrm{H}, J=5.0 \mathrm{~Hz}), 7.26$ (d, $1 \mathrm{H}$, $J=3.4 \mathrm{~Hz}), 7.06$ (t, $1 \mathrm{H}, J=4.3 \mathrm{~Hz}), 6.26(\mathrm{~d}, 1 \mathrm{H}, J=15.7 \mathrm{~Hz}), 4.21(\mathrm{t}, 2 \mathrm{H}, J=6.7 \mathrm{~Hz}), 1.70$ (quint, $2 \mathrm{H}, J=7.1 \mathrm{~Hz}$ ), 1.45 (sixet, $2 \mathrm{H}, J=7.5 \mathrm{~Hz}$ ), 0.98 (t, $3 \mathrm{H}, J=7.4 \mathrm{~Hz}$ ).

trans-Stilbene<smiles>C(=C/c1ccccc1)\c1ccccc1</smiles>

${ }^{1} \mathrm{H}$ NMR (500 MHz, $\left.\mathrm{CDCl}_{3}\right): \delta 7.55$ (d, $\left.4 \mathrm{H}, J=7.6 \mathrm{~Hz}\right), 7.40$ (t, $\left.4 \mathrm{H}, J=7.6 \mathrm{~Hz}\right), 7.30$ (t, $2 \mathrm{H}, J$ $=7.3 \mathrm{~Hz}), 7.15(\mathrm{~s}, 2 \mathrm{H})$

\section{2-Methyl-trans-stilbene}<smiles>[X]c1ccccc1/C=C/c1ccccc1</smiles>

${ }^{1} \mathrm{H}$ NMR (500 MHz, $\mathrm{CDCl}_{3}$ ): $\delta 7.64(\mathrm{~d}, 1 \mathrm{H}, J=7.4 \mathrm{~Hz}), 7.57$ (d, $\left.2 \mathrm{H}, J=7.8 \mathrm{~Hz}\right), 7.11-7.42$ (m, $7 \mathrm{H}), 7.04$ (d, $1 \mathrm{H}, J=16.2 \mathrm{~Hz}), 3.86(\mathrm{~s}, 3 \mathrm{H})$.

\section{4-Methyl-trans-stilbene}<smiles>Cc1ccc(/C=C/c2ccccc2)cc1</smiles>

${ }^{1} \mathrm{H}$ NMR (500 MHz, CDCl $): \delta 7.53$ (d, $\left.2 \mathrm{H}, J=7.7 \mathrm{~Hz}\right), 7.45(\mathrm{~d}, 2 \mathrm{H}, J=8.0 \mathrm{~Hz}), 7.38$ (t, $2 \mathrm{H}, J$ $=7.6 \mathrm{~Hz}), 7.28(\mathrm{t}, 1 \mathrm{H}, J=7.7 \mathrm{~Hz}), 7.20(\mathrm{~d}, 2 \mathrm{H}, J=7.9 \mathrm{~Hz}), 7.13(\mathrm{~d}, 1 \mathrm{H}, J=16.5 \mathrm{~Hz}), 7.09(\mathrm{~d}$, $1 \mathrm{H}, J=16.4 \mathrm{~Hz}), 2.39$ (s, $3 \mathrm{H}$ ).

\section{4-Methoxy-trans-stilbene}<smiles>COc1ccc(/C=C/c2ccccc2)cc1</smiles>

${ }^{1} \mathrm{H}$ NMR $\left(500 \mathrm{MHz}, \mathrm{CDCl}_{3}\right): \delta 7.52(\mathrm{~d}, 2 \mathrm{H}, \mathrm{d}, J=7.8 \mathrm{~Hz}), 7.48(\mathrm{~d}, 2 \mathrm{H}, J=8.6 \mathrm{~Hz}), 7.37$ (t, 2 $\mathrm{H}, J=7.5 \mathrm{~Hz}), 7.26$ (t, $1 \mathrm{H}, J=7.6 \mathrm{~Hz}), 7.10(\mathrm{~d}, 1 \mathrm{H}, J=16.3 \mathrm{~Hz}), 7.00(\mathrm{~d}, 1 \mathrm{H}, J=16.3 \mathrm{~Hz})$, $6.93(\mathrm{~d}, 2 \mathrm{H}, J=8.6 \mathrm{~Hz}), 3.86(\mathrm{~s}, 3 \mathrm{H})$.

\section{4-Formyl-trans-stilbene}


<smiles>O=Cc1ccc(/C=C/c2ccccc2)cc1</smiles>

${ }^{1} \mathrm{H}$ NMR (500 MHz, $\left.\mathrm{CDCl}_{3}\right): \delta 10.03(\mathrm{~s}, 1 \mathrm{H}), 7.90$ (d, $\left.2 \mathrm{H}, J=8.2 \mathrm{~Hz}\right), 7.69(\mathrm{~d}, 2 \mathrm{H}, J=8.2$ $\mathrm{Hz}), 7.58(\mathrm{~d}, 2 \mathrm{H}, J=7.5 \mathrm{~Hz}), 7.42(\mathrm{t}, 2 \mathrm{H}, J=7.6 \mathrm{~Hz}), 7.34(\mathrm{t}, 1 \mathrm{H}, J=7.4 \mathrm{~Hz}), 7.30(\mathrm{~d}, 1 \mathrm{H}, J$ $=16.3 \mathrm{~Hz}), 7.18(\mathrm{~d}, 1 \mathrm{H}, J=16.4 \mathrm{~Hz})$.

\section{4-Cyano-trans-stilbene}<smiles>N#Cc1ccc(/C=C/c2ccccc2)cc1</smiles>

${ }^{1} \mathrm{H}$ NMR (500 MHz, $\left.\mathrm{CDCl}_{3}\right): \delta 7.52-7.65(\mathrm{~m}, 6 \mathrm{H}), 7.42(\mathrm{t}, 2 \mathrm{H}, J=7.6 \mathrm{~Hz}), 7.35(\mathrm{t}, 1 \mathrm{H}, J=$ $7.4 \mathrm{~Hz}), 7.23(\mathrm{~d}, 1 \mathrm{H}, J=16.4 \mathrm{~Hz}), 7.11(\mathrm{~d}, 1 \mathrm{H}, J=16.3 \mathrm{~Hz})$.

\section{4-Trifluoromethyl-trans-stilbene}<smiles>FC(F)(F)c1ccc(/C=C/c2ccccc2)cc1</smiles>

${ }^{1} \mathrm{H}$ NMR (500 MHz, $\left.\mathrm{CDCl}_{3}\right): \delta 7.63$ (s, $\left.4 \mathrm{H}\right), 5.57$ (d, $\left.2 \mathrm{H}, J=7.5 \mathrm{~Hz}\right), 7.41(\mathrm{t}, 2 \mathrm{H}, J=7.6 \mathrm{~Hz})$, $7.33(\mathrm{t}, 1 \mathrm{H}, J=7.3 \mathrm{~Hz}), 7.23(\mathrm{~d}, 1 \mathrm{H}, J=16.4 \mathrm{~Hz}), 7.15(\mathrm{~d}, 1 \mathrm{H}, J=16.4 \mathrm{~Hz})$. 